\title{
New mass limit of white dwarfs
}

\author{
Upasana Das and Banibrata Mukhopadhyay
}

\author{
Department of Physics, Indian Institute of Science, Bangalore 560012, India \\ upasana@physics.iisc.ernet.in, bm@physics.iisc.ernet.in
}

June 6, 2018

\begin{abstract}
Essay received Honorable Mention in the Gravity Research Foundation 2013 Awards for Essays on Gravitation
\end{abstract}

\begin{abstract}
Is the Chandrasekhar mass limit for white dwarfs (WDs) set in stone? Not anymore - recent observations of over-luminous, peculiar type Ia supernovae can be explained if significantly super-Chandrasekhar WDs exist as their progenitors, thus barring them to be used as cosmic distance indicators. However, there is no estimate of a mass limit for these super-Chandrasekhar WD candidates yet. Can they be arbitrarily large? In fact, the answer is no! We arrive at this revelation by exploiting the flux freezing theorem in observed, accreting, magnetized WDs, which brings in Landau quantization of the underlying electron degenerate gas. This essay presents the calculations which pave the way for the ultimate (significantly super-Chandrasekhar) mass limit of WDs, heralding a paradigm shift 80 years after Chandrasekhar's discovery.
\end{abstract}

Keywords: white dwarfs; supernovae; stellar magnetic field; Landau levels; equation of state of gases

PACS Number(s): 97.20.Rp, 97.60.Bw, 97.10.Ld, 71.70.Di, 51.30.+i

\section{Introduction}

Chandrasekhar, in one of his celebrated papers [1], showed that the maximum possible mass of non-rotating, non-magnetized white dwarfs (WD) is $1.44 M_{\odot}$, when $M_{\odot}$ being the mass of Sun. He was awarded the Nobel Prize in Physics in 1983 mainly because of this discovery. This limiting mass (LM) is directly related to the luminosity of observed type Ia supernovae which are used as standard candles for measuring far away distances and hence in understanding the expansion history of the universe. The discovery of the accelerated expansion of the universe led to the Nobel Prize in Physics in 2011 [2]. 
So far, observations seemed to abide by the Chandrasekhar limit. However, in order to explain the recent discovery of several peculiar, anomalous, distinctly over-luminous type Ia supernovae [3, 4] - namely, SN 2006gz, SN 2007if, SN 2009dc, SN 2003fg - the mass of the exploding WDs (progenitors of supernovae) needs to be between $2.1-2.8 M_{\odot}$, significantly super-Chandrasekhar. Nevertheless, these non-standard 'super-Chandrasekhar supernovae' can no longer be used as cosmic distance indicators. However, there is need of a foundational level analysis, akin to that carried out by Chandrasekhar, in order to establish a super-Chandrasekhar mass WD. Moreover, there is no estimate of an upper mass limit for these super-Chandrasekhar WD candidates yet. Can they be arbitrarily large? These are some of the fundamental questions, we plan to resolve in the present essay.

\section{Basic physical process rendering the new limit}

We plan to exploit the effects of magnetic field to establish the new limit. Hence, we consider the collapsing star to be magnetized and the resulting accreting WD to be highly magnetized. This is in accordance with observations, which show that about $25 \%$ of accreting WDs, namely cataclysmic variables, are found to have surface magnetic fields as high as $10^{7}-10^{8} \mathrm{G}$ [5]. Hence their expected central fields could be $2-3$ orders of magnitude higher. If a magnetized WD gains mass due to accretion, its total mass increases which in turn increases the gravitational power and hence the WD contracts in size. However, the total magnetic flux in a WD, $\propto B R^{2}$, when $B$ is the magnetic field and $R$ the WD's radius, is conserved. Therefore, if the WD shrinks, its radius decreases and hence magnetic field increases. This in turn increases the outward force balancing the increased inward gravitational force, leading to a quasi-equilibrium situation. As accretion is a continuous process, the above process continues in a cycle and helps in increasing $B$ above the critical value $4.414 \times 10^{13} \mathrm{G}$ to bring in Landau quantization effects [6]. Subsequently, the mass of the WD keeps increasing, even above the Chandrasekhar limit, until the gain of mass becomes so great that it attains a new limit. At this point the total outward pressure is unable to support the gravitational attraction any longer, leading to a supernova explosion. This we argue to observe as a peculiar, over-luminous type Ia supernova, in contrast to their normal counter parts.

\section{Computing the new mass limit}

In the presence of strong magnetic field, the equation of state (EoS) of degenerate electron gas for WDs can be recast, at least in the piecewise zones of density $(\rho)$, in the polytropic form: $P=K_{m} \rho^{\Gamma}$, when $P$ is the pressure, $K_{m}$ and $\Gamma=1+1 / n$ are piecewise constants in different regimes of $\rho$ [6]. At the highest density regime (which also corresponds to the highest magnetic field regime), $\Gamma=2$. Now we recall the condition for magnetostatic equilibrium and estimate of mass, assuming the WD to be spherical, as

$$
\frac{1}{\rho} \frac{d}{d r}\left(P+\frac{B^{2}}{8 \pi}\right)=F_{g r}+\left.\frac{\vec{B} \cdot \nabla \vec{B}}{4 \pi \rho}\right|_{r}, \quad \frac{d M}{d r}=4 \pi r^{2} \rho,
$$

when $r$ is the radial distance from the center of WD and $F_{g r}$ the gravitational force. We 
note here that the choice of a Newtonian framework is justified in our case as the density corresponding to the degenerate pressure is much smaller than the matter density to contribute significantly to the effective mass of the WD (see Figure 1 of [6]). Moreover, in order to correctly include the effect of strong magnetic field in a general relativistic hydromagnetic balance equation, the Tolman-Oppenheimer-Volkov (TOV) equation, which is true only for the non-magnetic case, itself has to be modified first. Assuming $B$ varies very slowly around the center of the WD which is the regime of interest, we obtain [7]

$$
\frac{1}{r^{2}} \frac{d}{d r}\left(\frac{r^{2}}{\rho} \frac{d P}{d r}\right)=-4 \pi G \rho,
$$

where $G$ is Newton's gravitation constant. This further can be recast, with the use of EoS, into

$$
\frac{1}{\xi^{2}} \frac{d}{d \xi}\left(\xi^{2} \frac{d \theta}{d \xi}\right)=-\theta^{n} \text { with } \rho=\rho_{c} \theta^{n}
$$

where $\theta$ is a dimensionless variable and

$$
\xi=r / a, \quad a=\left[\frac{(n+1) K_{m} \rho_{c}^{\frac{1-n}{n}}}{4 \pi G}\right]^{1 / 2} .
$$

Equation (3) can be solved with the boundary conditions

$$
\theta(\xi=0)=1, \quad\left(\frac{d \theta}{d \xi}\right)_{\xi=0}=0 .
$$

Note that for $n<5, \theta$ becomes zero for a finite value of $\xi$, say $\xi_{1}$, which basically corresponds to the surface of the WD such that its radius

$$
R=a \xi_{1}
$$

Also the mass of the WD can be obtained as

$$
M=4 \pi a^{3} \rho_{c} \int_{0}^{\xi_{1}} \xi^{2} \theta^{n} d \xi .
$$

Now, the scalings of mass and radius of the WD with its central density $\left(\rho_{c}\right)$ are easily obtained as

$$
M \propto K_{m}^{3 / 2} \rho_{c}^{(3-n) / 2 n}, \quad R \propto K_{m}^{1 / 2} \rho_{c}^{(1-n) / 2 n}
$$

Clearly $n=3(\Gamma=4 / 3)$ corresponds to $M$ independent of $\rho_{c}$ (provided $K_{m}$ is independent of $\rho_{c}$ ) and hence LM. Therefore, we have to find out the condition for which $n=3$ and the corresponding proportionality constant for the scaling of $M$. Note, however, that $n=1$ for the extremely magnetized, highly dense, degenerate electron gas EoS, which is the present regime of interest. Below we explore the generic mass limit of WDs considering two scenarios. 
- Modeling WDs with varying magnetic field inside

Magnetized WDs are likely to have a varying $B$ profile, with an approximately constant field in the central region (CR), falling off from the central to surface region. As WDs evolve by accreting mass, their central and surface $B \mathrm{~s}$, along with the density, increase. This enables the WDs to hold more mass and hence they deviate from Chandrasekhar's mass-radius relation. Note that a large $B$ corresponds to a large outward magnetic pressure (along with a magnetic tension). Hence, an equilibrium solution depends on the nature of variation of $B$ within the WD such that it might no longer remain spherical. Moreover, how fast the field inside the WD decays to a smaller surface value affects its mass and radius. In order to give rise to a stable super-Chandrasekhar WD, the field needs to remain constant up to a certain region from the center, so that enough mass is accumulated due to the Landau quantized EoS. However, at the limiting (very large) density, when the mass becomes independent of (central) density, the basic trend of the mass-radius relation has to be same as that of Chandrasekhar, except for the larger mass, in order to achieve $n=3$ in EoS. At this situation, WDs will become, theoretically, very small such that $R=0$ (and hence the spherical assumption of its shape does not alter the result), as that obtained by Chandrasekhar in the absence of $B$. Hence, WDs close to the LM practically should have a constant $B$ throughout. However, at high density, $K_{m} \propto B^{-1} \propto \rho_{c}^{-2 / 3}$, unlike the non-magnetized EoS when $K_{m}$ is independent of $B$ (and $\left.\rho_{c}\right)$. Hence, when $\rho \rightarrow \rho_{c}$, the highly magnetized EoS reduces to

$$
P=K \rho^{4 / 3} \text { when } K=\frac{c \hbar \pi^{2 / 3}}{2^{1 / 3}\left(m_{H} \mu_{e}\right)^{4 / 3}},
$$

where $c$ is the speed of light, $\hbar$ the reduced Planck's constant, $m_{H}$ the mass of proton, $\mu_{e}$ the mean molecular weight per electron. Now combining equations (4), (77) for $K_{m}=K$ and $n=3$, we obtain the LM

$$
M_{l 1}=\frac{10.114}{\mu_{e}^{2}}\left(\frac{c \hbar}{G m_{H}^{4 / 3}}\right)^{3 / 2} .
$$

For carbon-oxygen WDs, $\mu_{e}=2$ and hence the LM becomes $4.67 M_{\odot}$.

- Modeling the central part of WDs

Here we consider only the CR of WDs and estimate the mass of this region. Of course the size of the CR changes as WDs evolve [8], which in turn determines how underestimated our result is with respect to the total mass (and total radius) of WDs. In CR 9 ]

$$
K_{m}=K \rho_{c}^{-2 / 3},
$$

and hence from equation (8)

$$
M \propto \rho_{c}^{3(1-n) / 2 n}, \quad R \propto \rho_{c}^{(3-5 n) / 6 n},
$$

revealing $M$ independent of $\rho_{c}$ for $n=1$, when the radius becomes independent of the mass in the mass-radius relation [8, 9]. Now combining equations (44), (7) with $n=1$, we 
obtain the value of LM

$$
M_{l 2}=\frac{5.564}{\mu_{e}^{2}}\left(\frac{c \hbar}{G m_{H}^{4 / 3}}\right)^{3 / 2} .
$$

For $\mu_{e}=2$ the LM becomes $2.58 M_{\odot}$. Note that $M_{l 2}$ is arrived at by considering a constant $B$ and hence is naturally smaller than $M_{l 1}$ which additionally counts the mass accumulated outside the $\mathrm{CR}$, for a varying $B$.

\section{Conclusions}

We summarize the findings of this work as follows:

- More than 80 years after the proposal of Chandrasekhar mass limit, this new limit perhaps heralds the onset of a paradigm shift.

- The masses of WDs are measured from their luminosities assuming Chandrasekhar's mass-radius relation, as of now. These results may have to be re-examined based on the new mass-radius relation, at least for some peculiar objects (e.g. over-luminous type Ia supernovae).

- Some peculiar known objects, like magnetars (highly magnetized compact objects, supposedly neutron stars, as of now), should be examined based on the above considerations, which could actually be super-Chandrasekhar WDs.

- This new mass limit should lead to establishing the underlying peculiar supernovae as a new standard candle for cosmic distance measurement.

- In order to correctly interpret the expansion history of the universe (and then dark energy), one might need to carefully sample the observed data from the supernovae explosions, especially if the peculiar type Ia supernovae are eventually found to be enormous in number. However, it is probably too early to comment whether our discovery has any direct implication on the current dark energy scenario, which is based on the observation of ordinary type Ia supernovae.

- Importantly, one now needs to carry out complete self-consistent calculations for the structure of WDs by generalizing the TOV equation to account for the strong magnetic field and pressure anisotropy, which has not been performed yet, in order to confirm the LM of WDs derived here.

\section{References}

[1] Chandrasekhar, S., MNRAS 95, 207 (1935).

[2] Perlmutter, S., et al., ApJ 517, 565 (1999).

[3] Howell, D.A., et al., Nature 443, 308 (2006).

[4] Scalzo, R.A., et al., ApJ 713, 1073 (2010). 
[5] Wickramasinghe, D.T., Ferrario, L., Pub. Ast. Soc. Pac. 112, 873 (2000).

[6] Das, U., Mukhopadhyay, B., Phys. Rev. D 86, 042001 (2012).

[7] Choudhuri, A.R., Astrophysics for Physicists. Cambridge Univ. Press, New York (2010).

[8] Das, U., Mukhopadhyay, B., Rao, A.R., ApJL 767, 14 (2013).

[9] Das, U., Mukhopadhyay, B., Phys. Rev. Lett. 110, 071102 (2013). 